\title{
ON A DOMAIN CHARACTERIZATION OF SCHRÖDINGER OPERATORS WITH GRADIENT MAGNETIC VECTOR POTENTIALS AND SINGULAR POTENTIALS
}

\author{
JEROME A. GOLDSTEIN AND ROMAN SVIRSKY
}

(Communicated by John B. Conway)

ABSTRACT. Of concern are the minimal and maximal operators on $L^{2}\left(\mathbf{R}^{n}\right)$ associated with the differential expression

$$
\tau_{Q}=\sum_{j=1}^{n}\left(i \partial / \partial x_{j}+q_{j}(x)\right)^{2}+W(x)
$$

where $\left(q, \ldots, q_{n}\right)=\operatorname{grad} Q$ for some real function $W$ on $\mathbf{R}^{n}$ and $W$ satisfies $c|x|^{-2} \leq W(x) \leq C|x|^{-2}$. In particular, for $Q=0, \tau_{Q}$ reduces to the singular Schrödinger operator $-\Delta+W(x)$. Among other results, it is shown that the maximal operator (associated with the $\tau_{Q}$ ) is the closure of the minimal operator, and its domain is precisely

$$
\operatorname{Dom}\left(\sum_{j=1}^{n}\left(i \partial / \partial x_{j}+q_{j}(x)\right)^{2}\right) \cap \operatorname{Dom}(W),
$$

provided that $C \geq c>-n(n-4) / 4$.

\section{INTRODUCTION}

Consider the formal differential expression

$$
\tau_{c}=H_{0}+V=-\Delta+c /|x|^{2}
$$

acting on functions on $\mathbf{R}^{n}$; here $\Delta$ is the Laplacian and $c$ is a real constant. The minimal and maximal operators associated with $\tau_{c}, H_{c m}$ and $H_{c M}$, are given by $\tau_{c}$ acting on the domains

$\mathscr{D}\left(H_{c m}\right)=C_{0}^{\infty}\left(\mathbf{R}^{n} \backslash\{0\}\right)=\left\{u \in C^{\infty}\left(\mathbf{R}^{n}\right): u\right.$ has compact support in $\left.\mathbf{R}^{n} \backslash\{0\}\right\}$, $\mathscr{D}\left(H_{c M}\right)=\left\{u \in L^{2}\left(\mathbf{R}^{n}\right): c|x|^{-2} u \in L_{\mathrm{loc}}^{1}\left(\mathbf{R}^{n}\right), \tau_{c} u \in L^{2}\left(\mathbf{R}^{n}\right)\right\}$.

Received by the editors February 16, 1988.

1980 Mathematics Subject Classification (1985 Revision). Primary 47B15, 47F05, 81C10.

Key words and phrases. Essential self adjointness, Schrödinger operator, singular potential, magnetic vector potential. 
(Here and in the sequel, " $m$ " stands for minimal and " $M$ " for maximal.) These operators, viewed as operators on $L^{2}\left(\mathbf{R}^{n}\right)$, have many remarkable properties. (Cf. [7]; see also [ 1,8$]$.) In particular we have:

(i) $H_{c m}$ is semibounded if and only if $H_{c m} \geq 0$ if and only if $c \geq$ $-[(n-2) / 2]^{2}$.

(ii) $H_{c m}$ is essentially selfadjoint if and only if $c \geq-n(n-4) / 4=1-$ $[(n-2) / 2]^{2}$.

(iii) $\overline{H_{c m}}=H_{c M}$ if and only if $c \geq-n(n-4) / 4$.

The remarkable aspect of (i)-(iii) is that these properties depend on the values of $c$ rather than the form of the potential. This is a hightly unusual occurrence in perturbation theory, and it shows that $c /|x|^{2}$ cannot be thought of as a small perturbation of $-\Delta$ if $c \neq 0$.

In our earlier paper [5] we extended these and other related results by taking advantage of scaling properties. More precisely, let $H_{0}=-\Delta, V(x)=c /|x|^{2}$, and $\lambda>0$. The unitary scaling operator $U(\lambda)$ is defined by

$$
(U(\lambda) f)(x)=\lambda^{n / 2} f(x) \text { for } f \in L^{2}\left(\mathbf{R}^{n}\right), x \in \mathbf{R}^{n} .
$$

Then

$$
U(\lambda) A U(\lambda)^{-1}=\lambda^{-2} A
$$

holds for both $A=H_{0}$ and $A=V$ (i.e., $A$ is multiplication by $V(x)$ ). Thus $-\Delta$ and $V(x)$ both scale like $\lambda^{-2}$. It turns out that the same is true of $\sum_{j=1}^{n}\left(i \partial / \partial x_{j}+\alpha x_{j} /|x|^{2}\right)^{2}$, and this fact formed part of the heuristic background for [5]. On the other hand, the vector whose $j$ th component is $\alpha x_{j}|x|^{-2}$ (for $\alpha \in \mathbf{R})$ is the gradient of $\alpha \log |x|$. Operators of the form

$$
\sum_{j=1}^{n}\left(i \partial / \partial x_{j}+q_{j}(x)\right)^{2}+c|x|^{2},
$$

where $\left(q, \ldots q_{n}\right)=\operatorname{grad} Q$, turn out to have the same properties as $H_{c m}$. Such a $\left(q, \ldots, q_{n}\right)$ will be termed a gradient magnetic vector potential. Properties of the operator (1) will be discussed in the sequel.

\section{BACKGROUND}

Clearly

$$
\mathscr{D}\left(H_{c M}\right) \supset \mathscr{D}\left(H_{0}\right) \cap \mathscr{D}(V) .
$$

It was recently discovered $[\mathbf{2}, \mathbf{6}, 12]$, that the converse containment holds if and only if $c>-n(n-4) / 4$. (Cf. also $[3,5,7,9,10]$.) Thus to (i)-(iii) we can add

(iv) $\mathscr{D}\left(H_{c M}\right)=\mathscr{D}\left(H_{0}\right) \cap \mathscr{D}(V)=\left\{u \in W^{2,2}\left(\mathbf{R}^{n}\right):|x|^{-2} u \in L^{2}(\mathbf{R})\right\}$ for

$$
c>c_{0}(n)= \begin{cases}\frac{3}{4} & \text { if } n=1 \text { or } 3, \\ 1 & \text { if } n=2, \\ 0 & \text { if } n \geq 4\end{cases}
$$


In particular, (iii) shows that (iv) holds only if $H_{c m}$ is essentially selfadjoint, in which case $\overline{H_{c m}}=H_{c M}$ is selfadjoint.

In fact, from [12] we can rewrite the criterion for (iv) in a more general form as follows.

Proposition 1. Let $H_{c M}, H_{0}$, and $V$ be as above. Then the following four statements are equivalent.

(a) $\mathscr{D}\left(H_{c M}\right)=\mathscr{D}\left(H_{0}\right) \cap \mathscr{D}(V)$.

(b) There exist constants $a \geq 0, b \geq 0$ such that

$$
\|V u\| \leq a\left\|H_{c M} u\right\|+b\|u\|
$$

holds for all $u \in \mathscr{D}\left(H_{c M}\right)$.

(c) There exists a constant $a \geq 0$ such that

$$
\|V u\| \leq a\left\|H_{c M} u\right\|
$$

holds for all $u \in \mathscr{D}\left(H_{c M}\right)$.

(d) $\mathscr{D}\left(H_{c M}\right) \subset \mathscr{D}(V)$ and $\left\|V\left(H_{c M}+I\right)^{-1}\right\|<\infty$.

In [5] we extended this to

$$
\tau_{c \alpha}=H_{0 \alpha}+V=\sum_{j=1}^{n}\left(i \partial / \partial x_{j}+\alpha x_{j} /|x|^{2}\right)^{2}+c /|x|^{2}
$$

(for $\alpha, c \in \mathbf{R}$ ) acting on functions on $\mathbf{R}^{n}$. As before, let $H_{c \alpha m}$ and $H_{c \alpha M}$ denote the minimal and maximal operators associated with $\tau_{c \alpha}$ on $L^{2}\left(\mathbf{R}^{n}\right)$, i.e.,

$$
\begin{gathered}
\mathscr{D}\left(H_{c \alpha m}\right)=C_{0}^{\infty}\left(\mathbf{R}^{n} \backslash\{0\}\right), \\
\mathscr{D}\left(H_{c \alpha M}\right)=\left\{u \in L^{2}\left(\mathbf{R}^{n}\right): c|x|^{-2} u \in L_{\mathrm{loc}}^{\prime}\left(\mathbf{R}^{n}\right)^{\prime}, \tau_{c \alpha} u \in L^{2}\left(\mathbf{R}^{n}\right)\right\} .
\end{gathered}
$$

Then Proposition 1 holds with $H_{c M}$ replaced by $H_{c \alpha M}$ for all $\alpha \in \mathbf{R}$.

In [5] we also pointed out the obstacle to (iv) holding for all positive $c$ in dimensions 2 and 3. Let $\mathscr{H}_{0}=L^{2}\left(\mathbf{R}^{n}\right)$ and let $\mathscr{H}_{1}$ be the closure in $\mathscr{H}_{0}$ of

$$
\left\{f \in L^{2}\left(\mathbf{R}^{n}\right) \cap C\left(\mathbf{R}^{n}\right): \int_{|x|=r} f(x) d S_{x}=0 \text { for each } r>0\right\},
$$

i.e. $\mathscr{H}_{1}$, consists of the functions in $\mathscr{H}_{0}$ having spherical means zero. For $l=0,1,2, \ldots$ let

$$
M_{l}=L^{2}([0, \infty)) \oplus H^{l}\left(S^{n-1}\right),
$$

so that the spherical harmonic decomposition of $L^{2}\left(\mathbf{R}^{n}\right)$ [13, p. 138ff.] becomes

$$
\mathscr{H}_{0}=L^{2}([0, \infty)) \otimes L^{2}\left(S^{n-1}\right)=L^{2}([0, \infty)) \otimes \bigoplus_{l=0}^{\infty} H^{l}\left(S^{n-1}\right) \equiv \bigoplus_{l=0}^{\infty} M_{l},
$$

and we have

$$
\mathscr{H}_{1}=M_{0}^{\perp}=\bigoplus_{l=1}^{\infty} M_{l}
$$


It was shown in [5] that a careful examination of the proof in [13] shows that for $c>0$ and $j=0,1$, there is a constant $a(j, n)$ such that

$$
\|V u\| \leq a(j, n)\left\|H_{c M} u\right\|
$$

holds for all $u \in \mathscr{D}\left(H_{c M}\right) \cap \mathscr{H}_{j}$ and all $c>c_{j}(n)$, where $c_{0}(n)$, is as before (in (iv)) and

$$
c_{1}(n)= \begin{cases}\frac{3}{4} & \text { if } n=1 \\ 0 & \text { if } n \geq 4 .\end{cases}
$$

Thus the obstruction to (iv) holding in dimension 2 and 3 is the subspace $M_{0}$ of radial functions.

\section{GRADIENT MAGNETIC VeCtOR POTENTIALS}

One of our goals here is to generalize the above results to the minimal and maximal operators, $H_{Q m}$ and $H_{Q M}$, associated with the differential expression

$$
\tau_{Q}=H(Q)+W=\sum_{j=1}^{n}\left(i \partial / \partial x_{j}+q_{j}(x)\right)^{2}+W(x)
$$

where $\left(q_{1}, \ldots, q_{n}\right)=\operatorname{grad} Q$ for some real function $Q$ in $W_{\text {loc }}^{1,1}\left(\mathbf{R}^{n}\right)$ and where the real function $W \in L_{\text {loc }}^{1}\left(\mathbf{R}^{n} \backslash\{0\}\right)$ satisfies $W(x) \geq c /|x|^{2}$ for all $x$ and some $c>-n(n-4) / 4$. The connection between $\tau_{Q}$ and the Schrödinger operator $-\Delta+W(x)$ is made clear by the following result.

Proposition 2. Let $A_{j}=i \partial / \partial x_{j}+q_{j}(x)$ for $j=1, \ldots, n$ where $\left(q_{1}, \ldots, q_{n}\right)=$ $\operatorname{grad} Q$ for some real function $Q$ in $W_{\mathrm{loc}}^{1,1}\left(\mathbf{R}^{n}\right)$. Let $U$ be the unitary operator of multiplication by $e^{i Q(x)}$. Then for $j \in\{1, \ldots, n\}, A_{j}=U B_{j} U^{-1}$ where $B_{j}=i \partial / \partial x_{j}$ acts on $\mathscr{D}\left(B_{j}\right)=\left\{u \in L^{2}\left(\mathbf{R}^{n}\right)\right.$ : the distributional derivative $\partial u / \partial x_{j}$ is in $\left.L^{2}\left(\mathbf{R}^{n}\right)\right\}$.

Proof. A straightforward computation gives

$$
U B_{j} U^{-1} u=\left(i \partial / \partial x_{j}+q_{j}(x)\right) u
$$

for any $u \in \mathscr{D}\left(A_{j}\right)=e^{i Q} \mathscr{D}\left(B_{j}\right)$.

The point of the above computation is that the unitary operator $U$ is independent of $j$.

Note that for $Q \equiv 0$, the minimal operator $H_{o m}$ is known to be essentially selfadjoint [11]. Also, $\bar{H}_{o m}=H_{o M}$. Combining these observations with the facts that $H(Q)=\sum_{j=1}^{n} A_{j}^{2}$ and $U W U^{-1}=W$ leads to the following result.

Corollary 3. Let $Q$ be as in Proposition 2. Then $H_{Q m}$ is essentially selfadjoint, $\overline{H_{Q m}}=H_{Q M}$, and $\mathscr{D}\left(H_{Q M}\right)=e^{i Q} \mathscr{D}\left(H_{o M}\right)$.

H. Kalf [6] conjectured that for suitable values of $c, \mathscr{D}\left(H_{o M}\right)=\mathscr{D}\left(H_{0}\right) \cap$ $\mathscr{D}(W)$. We shall establish a special case of this conjecture. Namely, we shall 
verify it for (measurable) potentials $W$ satisfying

$$
c_{1} /|x|^{2} \leq W(x) \leq c_{2} /|x|^{2}+c_{3}
$$

for any constants $c_{i}$ satisfying

$$
-n(n-4) / 4<c_{1} \leq c_{2}, \quad c_{3} \geq 0 .
$$

Theorem 4. Let (3) and (4) hold. Let $Q$ be as in Proposition 2. Then there exists a constant $a$, depending only on $c_{1}, c_{2}$ and $c_{3}$, such that

$$
\|W u\| \leq a\left\|H_{Q M} u\right\|+a\|u\|
$$

holds for all $u \in \mathscr{D}\left(H_{Q M}\right)$. Thus

$$
\mathscr{D}\left(H_{Q M}\right)=\mathscr{D}(H(Q)) \cap \mathscr{D}(W)
$$

and $H_{Q M}$ is selfadjoint.

Proof. It follows from the previous discussion that (5) holds if and only if

$$
\|W u\| \leq a\left\|H_{o M} u\right\|+b\|u\|
$$

holds for all $u \in \mathscr{D}\left(H_{O M}\right)$. Clearly (6) is equivalent to

$$
\left\|W\left(H_{o M}+I\right)^{-1}\right\|=\left\|W\left(H_{0}+W+1\right)^{-1}\right\|<\infty .
$$

Let $V(x)=c_{1} /|x|^{2}$. Then by Proposition 1 and (2) we have

$$
\left\|V\left(H_{c_{1} M}+I\right)^{-1}\right\|=\left\|V\left(H_{0}+V+1\right)^{-1}\right\|<\infty
$$

if and only if $c_{1}>-n(n-4) / 4$.

Note that $c_{1}$ can be negative only in dimension five or more. Define cutoff functions

$$
U_{\nu}(x)=U(x) \text { or } c_{1} \nu^{2}
$$

according as $|x| \geq 1 / \nu$ or $|x|<1 / \nu$, for $U=V, W$. Then $V_{\nu}$ and $W_{\nu}$ are in $L^{\infty}\left(\mathbf{R}^{n}\right)$, and $V_{\nu} \leq W_{\nu}$ holds on $\mathbf{R}^{n}$. The Trotter product formula (cf. e.g. $[4,8])$ implies that

$$
\exp \left\{-t\left(H_{0}+W_{n}\right)\right\} \phi \leq \exp \left\{-t\left(H_{0}+V_{n}\right)\right\} \phi
$$

for all $0 \leq \phi \in L^{2}\left(\mathbf{R}^{n}\right)$. Integrating this inequality gives the resolvent inequality

$$
\left(H_{0}+W_{n}+1\right)^{-1} \phi \leq\left(H_{0}+V_{n}+1\right)^{-1} \phi
$$

for all $0 \leq \phi \in L^{2}\left(\mathbf{R}^{n}\right)$. Letting $n \rightarrow \infty$ gives

$$
\left(H_{0}+W+1\right)^{-1} \phi \leq\left(H_{0}+V+1\right)^{-1} \phi
$$

for all such $\phi$.

From (3) we deduce, for all $0 \leq \phi \in L^{2}\left(\mathbf{R}^{n}\right)$,

$$
\begin{aligned}
|W|\left(H_{0}+W+1\right)^{-1} \phi \leq & \max \left(\left|c_{1}\right|,\left|c_{2}\right|\right)|x|^{-2}\left(H_{0}+W+1\right)^{-1} \phi \\
& +c_{3}\left(H_{0}+W+1\right)^{-1} \phi \\
\leq & \max \left(\left|c_{1}\right|,\left|c_{2}\right|\right)|x|^{-2}\left(H_{0}+V+1\right)^{-1} \phi \\
& +c_{3}\left(H_{0}+V+1\right)^{-1} \phi
\end{aligned}
$$

by (7). 
Next recall that for a bounded, positivity preserving operator $A$ we have $|A \psi| \leq A|\psi|$ a.e. for each $\psi \in L^{2}\left(\mathbf{R}^{n}\right)$. Thus we deduce

$$
\begin{aligned}
\left\|W\left(H_{0}+W+1\right)^{-1}\right\| \leq & \max \left(\left|c_{1}\right|,\left|c_{2}\right|\right)\left\||x|^{-2}\left(H_{0}+V+1\right)^{-1}\right\| \\
& +c_{3}\left\|\left(H_{0}+V+1\right)^{-1}\right\|<\infty .
\end{aligned}
$$

This completes the proof.

We remark that when $c_{1}>0$, the approximation argument (involving $V_{\nu}, W_{\nu}$ ) becomes unnecessary and the above proof simplifies.

The purpose of $c_{3}$ was to write $W$ as $W_{1}+W_{2}$ where

$$
c_{1} /|x|^{2} \leq W_{1}(x) \leq c_{2} /|x|^{2}, \quad W_{2} \in L^{\infty}\left(\mathbf{R}^{n}\right) .
$$

The bounded potential $W_{2}$ is a small perturbation of $H_{0}+W_{1}$ from the viewpoint of selfadjointness. Various unbounded potentials could take its place.

\section{ACKNOWLEDGMENTS}

We gratefully acknowledge the partial support of an NSF grant (J.G.) and a Faculty Research Fellowship from the University of Tennessee (R.S.). We have benefitted from helpful comments from several people, including Evans Harrell.

\section{REFERENCES}

1. P. Baras and J. A. Goldstein, Remarks on the inverse square potential in quantum mechanics, Differential equations (I. W. Knowles and R. T. Lewis, ed.), Elsevier (North-Holland), Dordrecht, 1984, pp. 31-35.

2. E. B. Davies, Some norm bounds and quadratic form inequalities for Schrödinger operators. II, J. Operator Theory 12 (1984), 177-196.

3. J. Glimm and A. Jaffe, Singular perturbation of self-adjoint operators, Comm. Pure Appl. Math. 22 (1969), 401-414.

4. J. A. Goldstein, Semigroups of linear operators and applications, Oxford Univ. Press, New York and Oxford, 1985.

5. J. A. Goldstein and R. Svirsky, Singular potentials and scaling, Houston J. Math. 13 (1987), 557-566.

6. H. Kalf, A note on the domain characterization of certain Schrödinger operators with strongly singular potentials, Proc. Roy. Soc. Edinburgh 97A (1984), 125-130.

7. H. Kalf, U.-W. Schmincke, J. Walter and R. Wüst, On the spectral theory of Schrödinger and Dirac operators with strongly singular potentials, Lecture Notes in Math., No. 448, SpringerVerlag, Berlin, 1975, 182-226.

8. M. Reed and B. Simon, Methods of modern mathematical physics. I, II, Academic Press, New York, 1972, 1976.

9. D. W. Robinson, Scattering theory with singular potentials. I, The two-body problem, Ann. Inst. H. Poincaré 21A (1974), 185-215.

10. D.-W. Schmincke, Essential self-adjointness of a Schrödinger operator with strongly singular potential, Math. Z. 123 (1972), 47-50.

11. B. Simon, Essential self-adjointness of Schrödinger operators with singular potential, Arch. Rational Mech. Anal. 52 (1973), 44-48. 
12. B. Simon, Hardy and Rellich inequalities in non-integral dimensions, J. Operator Theory 9 (1983), 143-146 and 12 (1984), 197.

13. E. M. Stein and G. Weiss, Introduction to Fourier analysis on Euclidean spaces, Princeton Univ. Press, Princeton, N.J., 1971.

Department of Mathematics, Tulane University, New Orleans, Louisiana 70118

Department of Mathematics, University of Tennessee, Knoxville, Tennessee 37996 